\title{
Germanica
}

\section{Interview de Hans-Georg Gadamer. Le dialogue herméneutique entre crise interpersonnelle et écriture (Heidelberg, 15 novembre 1994}

Hans-Georg Gadamer, Elfie Poulain et Jacques Poulain

\section{OpenEdition}

Journals

Édition électronique

URL : http://journals.openedition.org/germanica/1344

DOI : 10.4000/germanica.1344

ISSN : 2107-0784

Éditeur

Université de Lille

Édition imprimée

Date de publication : 30 juin 1998

Pagination : 183-193

ISBN : $9770984263203-22$

ISSN : 0984-2632

\section{Référence électronique}

Hans-Georg Gadamer, Elfie Poulain et Jacques Poulain, «Interview de Hans-Georg Gadamer. Le dialogue herméneutique entre crise interpersonnelle et écriture (Heidelberg, 15 novembre 1994 », Germanica [En ligne], 22 | 1998, mis en ligne le 30 janvier 2012, consulté le 06 octobre 2020. URL : http://journals.openedition.org/germanica/1344 ; DOI : https://doi.org/10.4000/germanica.1344

Ce document a été généré automatiquement le 6 octobre 2020.

(c) Tous droits réservés 


\title{
Interview de Hans-Georg Gadamer. Le dialogue herméneutique entre crise interpersonnelle et écriture (Heidelberg, 15 novembre 1994
}

\author{
Hans-Georg Gadamer, Elfie Poulain et Jacques Poulain
}

Ce texte constitue la version originale de l'interview d'H.G. Gadamer réalisée par Elfie et Jacques Poulain. Divers extraits en ont été publiés respectivement dans Le Monde du 3 janvier 1995 et dans la Frankfurter Rundschau du 11février 1995.

\section{Le concept d'herméneutique}

QUESTION : Votre œuvre philosophique est bien connue pour avoir développé ce que vous avez appelé dès le départ « herméneutique ». Même si le public cultivé a eu parfois du mal à comprendre ce qu'il faut entendre par là, l'herméneutique est devenue ces dernières décennies l'objet d'un intérêt de plus en plus soutenu au sein de la discussion philosophique internationale. Pouvez-vous nous préciser ce qui, à vos yeux, a fini par éveiller cet intérêt et nous expliquer pourquoi vous avez consacré votre œuvre au développement de l'herméneutique?

HANS-GEORG GADAMER : Bien entendu. La tâche que je m'étais fixée était au départ de cerner le concept d'herméneutique. J'en avais rencontré l'expression dans les écrits $\mathrm{du}$ romantisme allemand, puis dans les usages qu'en avait fait l'école phénoménologique avec Husserl et Heidegger en y voyant une nouvelle formule. Il faut en effet avoir toujours présent à l'esprit que le néo-kantisme qui dominait à cette époque, partait toujours d'un fait: celui de l'existence des sciences. C'était son premier et dernier argument. Je me rappelle avoir appris de mon maître Paul Natorp, professeur à Marbourg, la chose suivante : «Qu'est-ce que le donné ? Le donné est ce qui est à déterminer par les sciences. » Le débat philosophique tout entier s'en était vu ainsi extraordinairement rétréci et limité. C'est même encore visible dans le tournant de pensée qui s'est dessiné après la Première Guerre mondiale sous 
l'appellation d'existentialisme. Ce tournant constitua davantage une réaction et une riposte au néo-kantisme qu'un médium de pensée radicalement nouveau. Je suis devenu de plus en plus conscient de cette situation au furet à mesure que j'ai progressé dans mes propres recherches et au cours des rencontres que j'ai eu l'occasion de faire. Je me rappelle en particulier mon voyage à Mendoza, en Argentine, après la Seconde Guerre mondiale et la rencontre que j'y fis de collègues italiens, français et anglais après la longue période d'isolation dans laquelle nous nous étions trouvés en Allemagne. Ce qu'il me fut très intéressant de constater, c'était toute la masse de choses qu'on ne peut développer qu'à condition de parler à quelqu'un et d'avoir un réel échange avec lui. On jouit dans le dialogue d'une sorte d'avantage inaccessible à la pure et simple transmission d'un savoir monologique, qui n'advient qu'en imposant sa vérité. Cette conviction, voilà le secret d'un échange authentique : autrui ne me donne en retour que ce qui nous préoccupe tous deux. Cette idée était totalement inexistante dans l'Allemagne d'alors, sauf dans l'argumentation catholique et juive (je pense à Martin Buber) où l'on ne la faisait valoir que dans un style quasi-feuilletonesque. Mais dans les milieux académiques, il n'y avait, à vrai dire, rien de tel. De cet état de choses, il ne nous reste qu'une sorte de monument verbal. Il s'agit du terme de Vorlesung ${ }^{1}$.

C'est pratiquement incroyable pour nous aujourd'hui, mais il faut savoir se rendre compte, sans se sentir obligé d'en appeler tout de suite à McLuhan, de ce qu'a été la monologisation qu'a introduite le développement des sciences dans le monde occidental. Lorsque les mathématiques se sont libérées de l'envoûtement qu'elles exerçaient comme nouvelle rationalité pour devenir une sorte d'instrument de maîtrise de la nature, cela a constitué une sorte d'événement extraordinaire. Galilée, c'est cela. La science moderne réside en cela : elle tient à ce que le langage y soit devenu un instrument. Il s'y est produit ainsi bien entendu le contraire de ce que nous faisons lorsque nous nous entretenons en parlant.

\section{La critique herméneutique de la modernité}

aUestion: Peut-on aller jusqu'à dire que les déformations de la philosophie commencent avec cet engagement résolu de la modernité en faveur de la science?

H.G. GADAMER : Oui, c'est absolument exact. Nous devons toujours garder présent à l'esprit que nous parlons ainsi, somme toute, de conceptions abstraites du langage et que nous les avons acquises dans l'horizon du concept de science des temps modernes. Ces conceptions ne nous viennent pas de la parole et de la vie ellesmêmes. Si mon intérêt s'est porté vers la philosophie grecque, c'est pour ranimer, pour nous tous, les éléments positifs qui avaient disparu à la faveur de cette destruction scientiste de l'expérience de la communication. À la question de savoir ce qu'est l'herméneutique, je dirais que l'herméneutique réside avant tout à comprendre que nous ne trouvons jamais de mots qui parviendraient à exprimer quelque chose de définitif. Nous laissons donc toujours ouverte la suite qu'il faudrait donner à nos propos. Car là est l'essence du dialogue. Un dialogue n'a, en principe, aucune fin, mais de nouveaux éléments peuvent au contraire toujours surgir, et il peut toujours nous venir quelque chose de nouveau à l'esprit, ou, comme on dit encore en allemand, es kann einem etwas einfallen. Chaque idée nouvelle, chaque intuition subite est, en ce sens, une ouverture. Cette différence entre la conception 
instrumentale du langage et sa conception herméneutique est très profonde. Si je me suis rapproché autant de la philosophie grecque, ce n'est pas tellement à cause du dialogue socratique que je l'ai fait : celui-ci est déjà une forme littéraire où ces deux aspects se trouvent mêlés. Mais pensons, par exemple, à Aristote. J'y insiste toujours : ce ne sont pas des livres que nous lisons, mais nous sommes face à des matériaux de dialogues. Lorsque nous nous efforçons, en bons philologues, de dégager la position interne et définitive de la métaphysique aristotélicienne à partir de ces livres, nous nous donnons un point de départ complètement grotesque. Mais qu'en savons-nous? Quand Aristote exposait sa critique, incroyablement primitive, de Platon, voulait-il dire qu'il en était effectivement ainsi? Nous sommes beaucoup trop imprégnés de littérature pour comprendre ce que signifie, à lui seul, le fait qu'aucun homme de l'Antiquité, avant l'évêque de Milan (Saint-Ambroise), n'arrivait à lire sans parler à haute voix.

Question: La philosophie analytique a été longtemps indifférente à l'égard de I'herméneutique, voire même parfois hostile. Pourrait-on dire qu'elle est actuellement en train de changer d'attitude et de prêter au dialogue une attention toute nouvelle?

H.G. GADAMER:Vous en revenez, ajuste titre, à l'analogie qu'on retrouve dans l'évolution de la philosophie analytique et dans celle de l'herméneutique. Cela correspond tout à fait à l'expérience que j'ai faite à l'Université Queen's en Ontario, au Canada. Il y avait là un département composé au départ exclusivement de philosophes analytiques. L'un de mes anciens étudiants m'y a un jour invité et nous avons eu un bon débat. Il m'y a réinvité cinq ans plus tard. Étant donné mon âge et la fatigue qu'occasionne ce genre de voyages, je lui ai répondu que j'avais déjà eu l'occasion de connaître ses collègues. Mais il m'a répliqué : « Non, il n'en est rien, car ils sont tous devenus herméneutes, entre temps». Pourquoi? Ils avaient tout simplement compris que le même rapport puriste, le même effort de se libérer de toute construction théorique, était à l'œuvre dans la devise phénoménologique d'aller aux choses-mêmes et dans l'orientation qu'avait prise la philosophie analytique. Celle-ci s'était incroyablement rétrécie au point d'être incapable de rayonner sur l'ensemble de la culture. Cela ne signifie pas que la philosophie analytique ne traite pas de questions sérieuses. Ses questions sont sérieuses et elles ont persisté jusqu'à aujourd'hui, puisqu'on les importe actuellement en Allemagne et que cette importation y constitue ce qu'il y a de plus neuf. Je n'ai rien contre. Mais ce qui me semble le plus intéressant dans cette importation est qu'elle mette fin à l'isolation culturelle que l'Allemagne avait provoquée, car cette isolation signifiait aussi que tout venait de Hegel, qu'il était le seul coupable et que tout venait de la tradition idéaliste des Allemands. Je m'étais rendu compte par moi-même que la philosophie analytique avait des objectifs semblables à celle de cette tradition et que les frontières entre traditions devaient finir par disparaître. Mais nous, les Allemands, nous ne sommes malheureusement pas en mesure, et les Français non plus, de faire des tournures subtiles de la langue anglaise, l'objet de la philosophie. Pour y parvenir, il faudrait être tout à fait familier des expressions idiomatiques de cette langue.

\section{La poétique des langues}


QUESTION : La focalisation de l'analyse du langage sur la description des règles que nous sommes supposés y suivre, ne tend elle pas à faire abstraction de ce que vous appelez la dimension poétique des langues?

H.G. GADAMER : Cela ne fait aucun doute. Je crois en effet que ce qui libère le plus et qui est le plus vivant dans toutes les langues, c'est leur capacité poétique, leur capacité à susciter des intuitions qui nous parlent véritablement. Ce que j'ai compris dans la phénoménologie, et en premier lieu chez Husserl, c'est la chose suivante : il décrit et présente les choses les plus triviales avec une telle subtilité de langage qu'on a l'impression qu'on voit littéralement ce dont il est question. On n'a, à vrai dire, même plus besoin de ses mots.

On s'aperçoit de cela dès qu'on réfléchit à la façon dont la phénoménologie est entrée dans la littérature. On peut en effet malheureusement noter en ce domaine qu'on s'est pratiquement borné à en parler. Il n'y a guère eu d'effets véritables qui aient pu émaner de l'école phénoménologique pour la pure et simple raison que personne n'a su surmonter cette erreur d'appréciation du rôle de la philosophie. L'une des choses que je m'attribue, c'est d'avoir réussi à le faire par le biais de l'herméneutique.

\section{La question du bien et l'unité philosophique de la raison}

QUESTION : Vous parlez des déformations fondamentales qu'a subies la philosophie. En quoi consistent-elles au juste? Faut-il comprendre l'herméneutique comme une façon d'attirer l'attention sur ces déformations et de viser à en libérer? Est-ce ainsi qu'on doit lire votre ouvrage Vérité et méthode? ?

H.G. GADAMER : L'une des déformations déterminantes introduite par la modernité en philosophie réside dans la séparation de principe qui a été posée entre la raison théorique et la raison pratique. Celle-ci est apparue tout d'abord chez Aristote, chez un Aristote compris, bien entendu, de façon très scolaire. La problématique de Socrate était encore neutre en ce qui concerne cette séparation de la raison théorique et de la raison pratique. Mais si l'on se demande ce que les Grecs, et en particulier Socrate, avaient présent à l'esprit lorsqu'ils se posaient la question du bien, force est de constater que cette séparation n'y existe précisément pas. Percer l'ordre du monde, d'un côté, et s'efforcer, de l'autre, d'instaurer un ordre dans notre monde humain ne formaient ensemble qu'une seule et même tâche. Et c'est à cette tâche qu'il nous faut revenir pour surmonter toutes ces déformations dérivées de la modernité. C'est certainement l'un des points à propos desquels il m'a été donné d'apprendre quelque chose d'Aristote, bien qu'Aristote n'ait jamais abordé directement cette question pour elle-même. Il n'en a parlé qu'occasionnellement et indirectement, par exemple lorsqu'il se demande ce que font à vrai dire les dieux. Ces dieux étaient pour lui des "êtres purement théoriques ». Mais qu'est-ce qu'être « un être purement théorique » ? Cela signifie qu'ils s'adonnent de tout leur être à la tâche dans laquelle ils se plongent et qu'ils ont devant les yeux. Cela est d'une importance pratique considérable. Nous en faisons tous plus ou moins l'expérience lorsque nous nous sentons chercheurs. L'expression de "chercheur» est, elle aussi, un tant soit peu trompeuse, étant donné ses connotations géographiques et biographiques, qui nous donnent l'impression que des continents encore inconnus nous attendent toujours. Il n'en est pas véritablement ainsi, mais il y va quand même toujours de la 
question de savoir si nous sommes réellement capables de prendre au sérieux l'opinion d'autrui. Or n'était-ce pas ce dont il s'agissait d'abord et avant tout pour Hegel lorsqu'il cherchait à répondre à la question : qu'est-ce que la culture?

\section{Le dialogue interculturel dans le devenir du monde}

QUESTION : Les rencontres et les dialogues qui se multiplient de nos jours entre les différentes cultures de notre globe ne créent-ils pas de nouvelles conditions de compréhension mutuelle où peuvent se développer de nouvelles façons de penser?

H.G. GADAMER : Vous mettez le doigt sur ce que je considère comme le fin du fin de l'herméneutique. Car ce n'est pas forcément honteux qu'autrui pense autrement que moi. C'est même peut-être un gain. C'est même peut-être ainsi que nous nous ouvrons des horizons les uns aux autres. Je pense que la contribution minime apportée par chacun à la culture du monde réside en fin de compte en cela.

Il va de soi qu'on ne peut en faire directement un but final, et pourtant, dirais-je, l'Europe a encore beaucoup à apprendre pour être un jour en mesure d'établir ce genre de communauté de dialogue. Mais le monde dans son ensemble finira par le faire, car il sera contraint de le faire. Pour la première fois en effet, toutes les grandes cultures du monde, y compris celles qui sont issues des religions autres que la nôtre, se trouvent pour ainsi dire soudées les unes aux autres par l'économie mondiale. Aussi devons-nous apprendre à nous entendre désormais les uns avec les autres. Il n'en a jamais été ainsi. Nous admirons aujourd'hui le collègue japonais qui parvient à se retrouver dans le monde de nos concepts. Mais cela ne suffira pas, et de loin, à nous mener hors de notre langue et au-delà de nos propres barreaux conceptuels. Il pourra tout au plus, peut-être, nous donner en spectacle, de façon virtuose, la manière dont nous nous exprimons lorsque nous essayons de sortir de nous-mêmes sans parvenir à penser de façon productive. Et cela vaut bien entendu des deux côtés. Sortir de cette situation constituera à coup sûr une tâche pour le monde entier, si par bonheur, nous parvenons à apporter des solutions appropriées aux problèmes écologiques ainsi qu'à ceux que pose l'organisation du monde. Mais ce qu'il nous faudra avant tout apprendre de plus en plus, c'est qu'autrui est là, et que, lui aussi, il nous considère comme un autre. Nous devrons probablement accepter de plus en plus une coexistence réelle des différentes langues. Je me bats depuis toujours chez nous, en Allemagne, pour défendre l'idée qu'on devrait pouvoir parvenir soi-même à parler une deuxième langue si l'on veut pouvoir accéder à une compréhension là même où toute traduction échoue, c'est-à-dire dans le domaine de la poésie.

\section{L'aura de vérité propre à l'art poétique et à la communication}

QUESTION : Le dialogue recherché par l'herméneutique ne vise-t-il avant tout à faire partager cette force d'éveil de l'intuition, propre au langage et inhérente au premier chef à l'expérience poétique, parce que celle-ci nous fait toujours déjà surmonter la division, héritée de la modernité, entre raison cognitive, raison éthique et raison esthétique? La 
communication poétique ne demeure-t-elle pas, considérée sous cet angle, le modèle dont cherche à s'approcher toute communication?

H.G. GADAMER : C'est certain. Je pense, pour ma part, qu'il en est effectivement ainsi dès lors qu'on se sent, tant soit peu, chez soi dans une langue. J'ai toujours cherché effectivement à dégager la part de vérité inhérente à l'expérience du langage poétique et de l'art en général aussi bien qu'à l'herméneutique de la communication. Envisagée sous cet aspect, la musique, par exemple, constitue également l'une des promesses essentielles de l'avenir. Le monde asiatique, et, avec lui, le monde tout entier, s'est ouvert à la musique classique occidentale et vit dans cet univers.

C'est là un phénomène que je ne parviens pas, quant à moi, à comprendre dans toute son ampleur, et peut-être n'y a-t-il personne encore qui soit à même de le faire. Mais il y a plus encore. On ne se contente pas d'en vivre. Il va de soi qu'on cherche aussi à la dépasser en se lançant dans les aventures musicales les plus audacieuses. Le secret de tout art me semble résider là : ce qu'on y appelle « progrès » est toujours aussi une forme de retour. C'est ainsi que de nouvelles choses se révèlent constamment à nos yeux. Pensons, par exemple, à la sculpture africaine que nous avons appris à trouver magnifique au début de ce siècle. Si quelqu'un avait osé faire état de son admiration un siècle plus tôt, on l'aurait déclaré fou et enfermé dans un asile.

Le cas de la musique m'a particulièrement impressionné, mais j'admets volontiers qu'il en va de même pour la peinture. Paris m'a d'ailleurs donné l'occasion d'en faire l'expérience. Après bien des années, j'ai eu la chance, en 1993, d'y passer à nouveau quelques heures dans les musées. Et, comme cela arrive souvent à Paris, après avoir parcouru une collection extraordinairement riche, on en vient à déboucher sur les développements les plus récents de l'art pictural. On commence par se trouver surpris. On doit se reprendre pour s'en laisser imprégner, puis, lorsqu'on revient en arrière, c'est l'art antérieur qui nous fait à son tour l'effet d'être étrangement pâle. Tous ces chemins, on peut les parcourir dans un sens ou dans l'autre. Ce sont là les chemins de l'art, le privilège véritable de l'art. La simultanéité d'expérience qu'il sait instaurer à l'égard de lui-même acquiert ici une toute nouvelle valeur. Elle n'a quasiment d'égal que la valeur revendiquée en propre par la religion, elle a valeur de révélation.

QUESTION : Laissée à elle-même, la vie sociale, quotidienne peut devenir œuvre d'art, mais elle peut s'abîmer également dans les naufrages culturels qui sont devenus monnaie courante Iorsqu'on exploite aveuglément les conquêtes scientifiques et technologiques contemporaines à des fins contraires à l'éthique de la compréhension mutuelle. L'herméneutique n'est-elle pas impuissante à enrayer ces désastres de civilisation?

H.G. GADAMER : Ce destin propre à notre culture technologique et cette régulation technocratique qui s'étend de façon si puissante sur toute la planète, nous éveillent et nous sensibilisent pourtant en même temps à la liberté. Il existe à cet égard un très bel exemple, dont je me sers volontiers. Il m'est donné par l'ouvrage de Walter Benjamin intitulé L'Euvre d'art à l'heure de sa reproductivité technique ${ }^{3}$. L'auteur cherche à montrer que, dans le monde moderne, advient une nouvelle homogénéisation sociale. Celle-ci est liée à un nouvel ordre rationnel du monde social. Ce dernier, nous dit-il, ne pourra plus désormais se produire qu'en se reproduisant puisque l'aura propre à l'art y disparaîtra. Voilà une magnifique prophétie du contraire de ce qui arrive, car ce que nous voyons, c'est que nous ressentons à nouveau cette aura. Partout on voit les gens se précipiter devant les originaux, malgré toute l'invasion 
des reproductions qu'on peut constater. Cette aura du se comprendre l'un l'autre, voilà la nouvelle bonne nouvelle. Voyez ces visiteurs qui s'attardent un bon quart d'heure devant le tableau de Guernica. Pourquoi resteraient-ils là un quart d'heure si ce n'est parce qu'ils ressentent cette aura? Pourquoi s'attarderaient-ils s'ils ne pressentaient pas que, même ce qu'il y a de fragmentaire dans notre vie, peut parvenir à s'énoncer grâce à notre faculté de compréhension herméneutique?

J'ai moi-même fait l'expérience, partout où j'étais, de l'extrême importance qu'il y a à garder cette ouverture. On ne la maintient vraiment qu'en devenant conscient de l'incapacité radicale dans laquelle on sera toujours d'avoir le dernier mot. Voilà finalement en quoi consiste l'herméneutique. Elle consiste à savoir cette chose infiniment simple : le dernier mot, eh bien, non, je ne veux pas l'avoir.

\section{NOTES}

1. Note du traducteur : Le terme Vorlesung signifie « leçon », « cours magistral », mais son sens étymologique renvoie à l'acte de lire un discours devant un auditoire.

2. Cf. H.G. Gadamer, Vérité et méthode, Paris, Ed. du Seuil, Trad. E. Sacre et P. Ricœur, 1973. Nouvelle traduction de P. Fruchon et J. Grondin, Paris, Ed. du Seuil, 1995.

3. La traduction française de l'ouvrage est due à $\mathrm{M}$. de Gandillac in L'homme, le langage, les cultures, Paris, Denoël, 1971, p. 145 et sv. 